\section{Response to 'Titre-specific positive predictive value of anti-nuclear antibody patterns' by Vulsteke et al}

Recently, a review on the clinical relevance of human epithelial (HEp)-2 indirect immunofluorescence assay (IIFA) patterns was published by the International Consensus on ANA Patterns (ICAP) consortium. ${ }^{1}$ The clinical relevance was primarily defined within the context of the clinical manifestations, that is, the suspected disease, and included recommendations for follow-up or reflex testing. It is anticipated that if a solid-phase test identifies the disease-specific target autoantigen, the clinical relevance can be further refined. The analyses by Vulsteke $e t a l^{2}$ elegantly illustrate that the positive predictive value (PPV) of the HEp-2 IIFA pattern can be further improved by taking into account the titre of the respective patterns. Given the value and importance of the large dataset $(n=9851)$ obtained in this routine laboratory practice without any selection, except for studying only monospecific nuclear patterns, and the extensive analyses, it is unfortunate that the results have been condensed into a letter. In addition to the patient cohort from the University Hospitals Leuven, a smaller cohort $(n=529)$ was analysed. In this second cohort from the OLV Hospital Aalst, sera with a nuclear speckled pattern $(n=88)$ were included; only four sera were obtained from patients with a systemic autoimmune rheumatic disease (SARD). Evidently, only the large dataset warranted reliable associations between the combination of HEp-2 IIFA pattern and titre on the one hand and the final diagnosis on the other.

With respect to the ICAP consensus on the clinical relevance of HEp-2 IIFA patterns, there are three deviations from this consensus as reported in the study of Vulsteke et al. First, the genetically modified HEp-2000 substrate was used, whereas this substrate is currently not included in the ICAP consensus for reasons summarised in a recent ICAP correspondence. ${ }^{3}$ As stated in this correspondence, the ICAP decision to exclude the HEp-2000 substrate might be reconsidered, although a previous study observed that some patterns are not readily recognised in this cell line. ${ }^{4}$ Importantly, the SS-A/Ro60 pattern observed on the transfected HEp-2 cell line will reduce the PPV of the fine speckled pattern (AC-4) with anti-SS-A/Ro60 antibody-associated diseases. Indeed, the SS-A/Ro60 pattern was frequently observed in systemic lupus erythematosus (SLE; $n=22$ ), Sjögren's syndrome $(S j S ; n=27)$ and cutaneous lupus erythematosus $(n=9)$. Second, only four nuclear patterns were included in the report: homogeneous (AC-1), centromere (AC-3), speckled (AC-4/AC-5) and nucleolar (AC-8/AC-9/AC-10). This simplified pattern classification likely will result in lower PPVs than would be achieved with full pattern classification. For instance, when using the HEp-2 substrate, the SS-A/Ro60 reactivity could be attributed to the AC-4 pattern, while U1-RNP reactivity could be attributed to the AC-5 pattern. By combining both patterns, U1-RNP reactivity would reduce the PPV for SjS, while SS-A/ Ro60 reactivity would reduce the PPV for mixed-connective tissue disease. Although currently not included in the ICAP classification, Luis Andrade recently presented observations on the HEp-2 substrate that suggested further differentiation within the AC-4 pattern grouping, distinguishing two AC-4 pattern variants with distinct association with anti-SS-A/Ro60 antibodies (14th Dresden Symposium on Autoantibodies). Another limitation of the restricted pattern assignment in the study of Vulsteke et $a l^{2}$ is the exclusion of the cytoplasmic patterns as it would have been interesting to see how the titre contributes to the clinical interpretation of the cytoplasmic speckled patterns (AC-18, AC-19 and AC-20). In particular, the antisynthetase antibodies reveal only low to moderate titres. Third, the nuclear dense fine speckled pattern (AC-2) is only partially integrated in the study of Vulsteke et al. Similar to the HEp-2000 substrate, also the dense fine speckled pattern has been the topic of a recent ICAP correspondence. ${ }^{5}$ Importantly, the clinical relevance of the dense fine speckled pattern is defined by the correct recognition of the HEp-2 IIFA pattern, which is prone to interobserver variability, ${ }^{67}$ in combination with confirmation for being monoreactive with DFS70. In the study of Vulsteke et $a l^{2}{ }^{2}$ the dense fine speckled pattern was not identified as such but was primarily included in the homogeneous pattern (AC-1) and was retrospectively evaluated by analysing all sera with the homogeneous pattern with a titre of $\geq 1 / 320(n=163)$ and negative for anti-dsDNA antibodies and/or the connective tissue disease (CTD) screen $(n=93)$ for anti-DFS70 antibodies. About $20 \%$ of the sera with a medium-titre/high-titre homogeneous pattern $(n=35)$ appeared to be monospecific for DFS70. Thirty-three of the respective patients did not have a SARD. Although Vulsteke et $a l^{2}$ clearly illustrate that exclusion of samples with a suspected dense fine speckled pattern may not have a strong effect on the PPV, this pattern appears to have a high negative predictive value (NPV) for SARD (94\%). As recognised by the authors, this in particular holds for sera with medium/high HEp-2 IIFA titres ${ }^{89}$ and therefore draws into question the overall conclusion that high titres are better associated with SARD. The observed high NPV for SARD, however, is clinically relevant because the HEp-2 IIFA is often used to exclude the diagnosis of SARD, in particular, SLE and systemic sclerosis (SSc). These observations indicate that the nuclear dense fine speckled pattern, therefore, may extend the NPV of the HEp-2 IIFA beyond the so-called 'negative' result.

A second issue that may be worthy of discussion is whether the titre is the driving factor for the increased PPV of the included HEp-2000 IIFA patterns. The authors acknowledge that the level of the autoantibodies is associated with increased probability of a final diagnosis within the spectrum of SARD, but also that there is an association with specific autoantibodies in the reflex testing. In the study of Vulsteke et $a{ }^{2}{ }^{2}$ the highest PPVs for SARD were observed for the centromere pattern (AC-3) and the SS-A/Ro60 pattern on the the HEp-2000 substrate. Although the titres observed may be different for distinct IIFA patterns, both the centromere and SS-A/Ro60 patterns typically present with high titres and reveal specific autoantibodies in reflex testing, that is, 93\% and 98\%, respectively, as in the study of Vulsteke et $a l^{2}$ The authors tried to tackle this enigma by examining if the increased PPV was also observed in sera that tested negative in the Farr assay and CTD screen (supplemental data). The prevalence of such conditions was somewhat higher for speckled and nucleolar patterns, and included patients with SLE, SSc and idiopathic inflammatory myopathies. Therefore, it can be speculated that these samples are reactive with Ku or TIF1 $\gamma$ (speckled pattern) or with NOR90 or Th/To (nucleolar pattern); none of these antigens were included in the CTD screen. Altogether, it remains to be established if the titre is the driving force or a contributing factor in the increased diagnostic value for individual patterns.

In conclusion, the study of Vulsteke et $a l^{2}$ illustrates that the clinical relevance of HEp-2 IIFA patterns can be improved by including the titre in the calculation of the PPV for SARD. Taking into account that PPV and NPV are parameters dependent on the prevalence of the disease and that this may differ between geographical areas, the conclusion that combining HEp-2 IIFA pattern and titre will increase the PPV can be generalised, but this 
does not necessarily hold for the values calculated in the patient cohort from Belgium. Here, we argue that the PPV can even be further increased, as well as the NPV in case of the nuclear dense fine speckled pattern (AC-2), by considering the full ICAP HEp-2 IIFA pattern classification. Eventually, we fully agree with the statement that confirmatory antigen-specific immunoassays are mandatory in all cases, preferentially by taking into account the diagnostic suspicion at the time of presentation, the centromere pattern (AC-3) being the only possible exception.

\section{Jan Damoiseaux $\odot,{ }^{1}$ Luís Eduardo Coelho Andrade, ${ }^{2}$ Marvin J Fritzler, ${ }^{3}$ Manfred Herold, ${ }^{4,5}$ Maria Infantino $\odot{ }^{6}$ Carlos von Muhlen ${ }^{7}$ \\ ${ }^{1}$ Central Diagnostic Laboratory, Maastricht University Medical Center, Maastricht, The Netherlands \\ ${ }^{2}$ Immunology, Fleury Laboratory, São Paulo, Brazil \\ ${ }^{3}$ Medicine, Health Sciences Centre, Calgary, Alberta, Canada \\ ${ }^{4}$ Department of Internal Medicine II, Innsbruck Medical University, Innsbruck, Austria \\ ${ }^{5}$ Department of Internal Medicine II, Tirol Kliniken GmbH, Innsbruck, Austria \\ ${ }^{6}$ Laboratory of Immunology and Allergology, Ospedale San Giovanni di Dio, Firenze, Italy \\ ${ }^{7}$ Brazilian Society of Autoimmunity, Porto Alegre, Brazil}

Correspondence to Dr Jan Damoiseaux, Central Diagnostic Laboratory, Maastricht University Medical Center, Maastricht 6229 HX, Netherlands;

jan.damoiseaux@mumc.nl

Handling editor Josef S Smolen

Acknowledgements The authors acknowledge the contribution of all members of the International Consensus on ANA Patterns Executive Board, including Orlando Gabriel Carballo, Paulo Luis Carvalho Francescantonio, Edward Chan, Karsten Conrad, Ignacio Garcia de la Torre, Werner Klotz, Wilson de Melo Cruvinel, Tsuneyo Mimori, Lucile Musset and Minoru Satoh.

Contributors All authors were involved in the design of the response; JD wrote the response, which was commented on by the other authors. Remarks and suggestions were included by JD.

Funding The authors have not declared a specific grant for this research from any funding agency in the public, commercial or not-for-profit sectors.

Competing interests None declared.

Patient consent for publication Not required.

Provenance and peer review Commissioned; internally peer reviewed.

(c) Author(s) (or their employer(s)) 2019. No commercial re-use. See rights and permissions. Published by BMJ.

\section{Check for updates}

To cite Damoiseaux J, Andrade LEC, Fritzler MJ, et al. Ann Rheum Dis Epub ahead of print: [please include Day Month Year]. doi:10.1136/annrheumdis-2019-216266

Received 20 September 2019

Accepted 25 September 2019

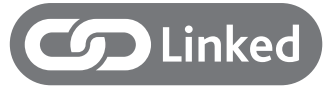

http://dx.doi.org/10.1136/annrheumdis-2019-216245

Ann Rheum Dis 2019;0:1-2. doi:10.1136/annrheumdis-2019-216266

\section{ORCID iDs}

Jan Damoiseaux http://orcid.org/0000-0003-4007-6985

Maria Infantino http://orcid.org/0000-0002-6200-4467

\section{REFERENCES}

1 Damoiseaux J, Andrade LEC, Carballo OG, et al. Clinical relevance of HEp-2 indirect immunofluorescent patterns: the International consensus on ANA patterns (ICAP) perspective. Ann Rheum Dis 2019;78:879-89.

2 Vulsteke JB, Van Hoovels L, Willems P, et al. Titre-specific positive predictive value of anti-nuclear antibody patterns. Ann Rheum Dis.

3 Damoiseaux J, Chan EK. Response to: 'The utility of the HEp-2000 antinuclear antibody substrate' by Lee et al. Ann Rheum Dis 2019:annrheumdis-2019-215610.

4 Dellavance A, Cruvinel WdeM, Francescantonio PLC, et al. Variability in the recognition of distinctive immunofluorescence patterns in different brands of HEp-2 cell slides. J. Bras. Patol. Med. Lab. 2013;49:182-90.

5 Andrade LEC, Damoiseaux J, Chan EK. Response to 'Decision making value of nuclear dense fine speckled pattern in systemic autoimmune rheumatic disease: trick or treat?' by Deng et al. Ann Rheum Dis 2019:annrheumdis-2019-215640.

6 Bentow C, Fritzler MJ, Mummert E, et al. Recognition of the dense fine Speckled (DFS) pattern remains challenging: results from an international Internet-based survey. Autoimmun Highlights 2016;7.

7 Rigon A, Infantino M, Merone M, et al. The inter-observer reading variability in antinuclear antibodies indirect (ANA) immunofluorescence test: a multicenter evaluation and a review of the literature. Autoimmun Rev 2017;16:1224-9.

8 Mariz HA, Sato El, Barbosa SH, et al. Pattern on the antinuclear antibody-HEp-2 test is a critical parameter for discriminating antinuclear antibody-positive healthy individuals and patients with autoimmune rheumatic diseases. Arthritis Rheum 2011;63:191-200

9 Carbone T, Pafundi V, Tramontano G, et al. Prevalence and serological profile of antiDFS70 positive subjects from a routine ANA cohort. Sci Rep 2019;9:2177. 